\title{
Intuitionistic Fuzzy Translation INK-ideal in INK-algebra
}

\author{
M. Kaviyarasu, K. Indhira
}

\begin{abstract}
The ideas of intuitionistic fuzzy translation in INKI's in INK-algebras are presented. The view of intuitionistic fuzzy extensions $\left(I F_{-} E\right)$ and $\left(I F_{-} M\right)$ intuitionistic fuzzy multiplications of fuzzy INK-ideals per more than a few related assets are examined. As well, the associations sandwiched between IF translations, IF_E and IF_M of IF_INKIs are studied.
\end{abstract}

Key words. INK-algebra, IF_INK-ideal, IF_translation, IF extension, $I F_{-}$multiplication.

\section{INTRODUCTION}

Lee et al. as well Jun deliberated $\mathrm{F}_{-}$translations, $\mathrm{F}_{-}$ extensions too $\mathrm{F}_{-}$multiplications of $\mathrm{F}_{-}$sub algebras in addition ideals in BCK/BCI-algebras. They well-thought-out kindred mid $F_{-} T, F_{-} E$ and $F_{-}$M. In this paper, $I_{-}$ translations, IF_ E and $\mathrm{IF}_{-} \mathrm{M}$ of $\mathrm{F}_{-}$INK-I in INK-algebras stay conferred. Relatives midst $\mathrm{IF}_{-}$translations, $\mathrm{IF}_{-} \mathrm{M}$ and IF_M of FINK_I in INK-algebras stand as well examined.

\section{PRELIMINARIES}

An algebra $(K \dot{K} ; \diamond, 0)$ is named a INK-algebra uncertainty it mollifies the ensuing situations,

- $((\mathrm{x} \diamond \square) \diamond(\mathrm{x} \diamond \mathrm{z})) \diamond(\mathrm{r} \diamond \square)=0$

- $((\mathrm{x} \diamond \mathrm{z}) \diamond(\square \diamond \mathrm{z})) \diamond(\mathrm{x} \diamond \square)=0$

- $\mathrm{x} \diamond 0=\mathrm{x}$

- $\mathrm{x} \diamond \square=0$ and $\square \diamond \mathrm{x}=0$ imply $\mathrm{x}=\square$, for all $\mathrm{x}, \square, \mathrm{z}$ in $\mathrm{K}$

$A F_{S} \lambda$ in a INK-algebra $K$ is named a $F_{-}$INK-subalgebra of $K$, if

$\lambda(\mathrm{x} \diamond \mathrm{y}) \geq \min \{\lambda(\mathrm{x}), \lambda(\mathrm{y})\}, \forall \mathrm{x}, \mathrm{q} \in \mathrm{K}^{\circ}$.

Let $(\hat{K}, \diamond, 0)$ be a INK-algebra. A subclass $\square$ of $K$ is named a INK-subalgebra of $K^{\prime}$ if the constant 0 of $K^{\prime}$ is in $\square$, and ( $\square$, $\diamond, 0)$ itself procedures a INK-algebra. if,

$A F_{S} \lambda$ in a INK-algebra $K$ is named a $F_{-}$INK- ideal of Ḱ,

- $\lambda(0) \geq \lambda(x)$

$\lambda(\mathrm{x}) \geq \min \{\lambda(\mathrm{z} \diamond \mathrm{x}) \diamond(\mathrm{z} \diamond \square), \lambda(\square)\}, \forall \mathrm{x}, \square, \mathrm{z}$ in $\mathrm{K}$.
Definition 2.1 An IF set $A$ in a non-void set $\dot{K}$ is an object having the form $A=\left\{\left(x, \lambda_{A}(x), \xi_{A}(x)\right) / x \in K\right\}$, where $\lambda_{A}: \dot{K} \rightarrow[0,1]$ and $\xi_{A}: \dot{K} \rightarrow[0,1]$ denote (namely $\left.\lambda_{A}(x)\right)$ the degree of membership and (namely $\left.\xi_{A}(x)\right)$ the degree of non-membership of $\dot{K}$ to the set $A$ correspondingly, and $0 \leq \lambda$ ${ }_{A}(x)+\xi_{A}(x) \leq 1$, for all $x \in K$ K. For the take simplicity, then $A=$ $\left(x, \lambda_{A}, \xi_{A}\right)$ for the $I F_{-}$set $A=\left\{\left(x, \lambda_{A}(x), \xi_{A}(x)\right) / x\right.$ in $\left.\dot{K}\right\}$.

Definition 2.2 An IF set $A=\left(x, \lambda_{A}, \xi_{A}\right)$ is named an $I F_{-}$ sub algebra of $K$ if it satisfies,

i) $\quad \lambda_{\mathrm{A}}(\mathrm{x} * \square) \geq \min \left\{\lambda_{\mathrm{A}}(\mathrm{x}), \lambda_{\mathrm{A}}(\square)\right\}$,

ii) $\quad \xi_{\mathrm{A}}\left(\mathrm{x}^{*} \square\right) \leq \max \left\{\xi_{\mathrm{A}}(\mathrm{x}), \xi_{\mathrm{A}}(\square)\right\}$, for all $\mathrm{x}, \square$ in K.

\section{MAIN RESULTS}

\subsection{Intuitionistic Fuzz $\square \tilde{\alpha}$-Translation of IFINK-ideal.}

Let $\mathrm{A}=\left(\lambda_{\mathrm{A}}, \xi_{\mathrm{A}}\right)$ for the IF_S is denoted by $\mathrm{A}=$ $\left\{\left\langle x, \lambda_{A}(\mathrm{x}), \xi_{A}(\mathrm{x}) \mid \mathrm{x} \in \mathrm{K}\right\rangle\right\}$. We yield $\mathrm{T}=1-\inf$ $\left\{\xi A\left(x_{1}\right) \mid p\right.$ in $\left.\dot{K}\right\}$ for any $A=\left(\lambda_{A}, \xi_{A}\right)$ of $\dot{K}$.

Definition 3.1.1

Let $A=\left(\lambda_{\mathrm{A}}, \xi_{\mathrm{A}}\right)$ be an IFS of Ḱ \& let $\tilde{\alpha}$, in [0, T]. An object having the form

$A_{\alpha}{ }^{T}=\left(\left(\lambda_{\mathrm{A}}\right)_{\alpha}{ }^{T},\left(\xi_{\mathrm{A}}\right)_{\alpha}{ }^{T}\right) \quad$ is $\quad$ named an IF- $\tilde{\alpha}$-translation of $\mathrm{A}$ if $\left(\lambda_{\mathrm{A}}\right)_{\alpha}{ }^{T}(x)=\lambda_{A}\left(\mathrm{x}_{\mathrm{A}}\right)+\alpha$ and

$\left(\xi_{\mathrm{A}}\right)_{\alpha}{ }^{T}(x)=\xi_{A}(\mathrm{x})-\alpha$, for every $\mathrm{x}$ in $\mathrm{K}$.

Theorem.3.1.2 Let $\mathrm{A}=\left(\lambda_{\mathrm{A}}, \xi_{A}\right)$ is an IFINK-ideal of $\dot{K}$, the $\mathrm{IF}_{-} \tilde{\alpha}_{-}$translation $A_{\tilde{\alpha}}^{T}$ of $\mathrm{A}$ is a IFINK-sub algebra of K.

Proof. Let $\mathrm{x}, \square \varepsilon \dot{\mathrm{K}}$,

$\left(\lambda_{A}\right)_{\tilde{\alpha}}^{T}(x \diamond \square)=\lambda_{A}(x \diamond \square)+\tilde{\alpha}$

$=\min \left\{\lambda_{A}(\mathrm{z} \diamond(\mathrm{x} \diamond \square)) \diamond(\mathrm{z} \diamond \square), \lambda_{A}(\square)\right\}+\tilde{\alpha}$

$\left.=\min \left\{\lambda_{A}((\mathrm{x} \diamond \square)) \diamond \square\right), \lambda_{A}(\square)\right\}+\tilde{\alpha}$

$=\min \left\{\lambda_{A}(0), \lambda(\square)\right\}+\tilde{\alpha}$

$\geq \min \left\{\lambda_{A}(x), \lambda(\square)\right\}+\tilde{\alpha}$

$$
\left(\lambda_{A}\right)_{\tilde{\alpha}}^{\mathrm{T}}(\mathrm{x} \diamond \square) \geq \min \left\{\left(\lambda_{\mathrm{A}}\right)_{\tilde{\alpha}}^{\mathrm{T}}(\mathrm{x}),\left(\lambda_{\mathrm{A}}\right)_{\tilde{\alpha}}^{\mathrm{T}}(\square)\right\}
$$

And

$\left(\xi_{A}\right)_{\tilde{\alpha}}^{T}(\mathrm{x} \diamond \square)=\xi_{A}(\mathrm{x} \diamond \square)+\tilde{\alpha}$

$=\max \left\{\xi_{A}(z \diamond(\mathrm{x} \diamond \square)) \diamond(\mathrm{z} \diamond \square), \xi_{A}(\square)\right\}-\tilde{\alpha}$

$\left.=\max \left\{\xi_{A}((\mathrm{x} \diamond \square)) \diamond \square\right), \xi_{A}(\square)\right\}-\tilde{\alpha}$

$=\max \left\{\xi_{A}(0), \xi_{A}(\square)\right\}-\tilde{\alpha}$

$\leq \max \left\{\xi_{A}(\mathrm{x}), \xi_{A}(\square)\right\}-\tilde{\alpha}$

$$
\left(\xi_{A}\right)_{\tilde{\alpha}}^{T}(\mathrm{x} \diamond \square) \leq \max \left\{\left(\xi_{A}\right)_{\tilde{\alpha}}^{T}(\mathrm{x}),\left(\xi_{A}\right)_{\tilde{\alpha}}^{T}(\square)\right.
$$

Revised Version Manuscript Received on 10 September, 2019.

M. Kaviyarasu, Research Scholar, Department of Mathematics, VIT University, Vellore, Tamilnadu, India. (Email: kavitamilm@gmail.com)

K. Indhira, Associate Professor, Department of Mathematics, VIT University, Vellore, Tamilnadu, India. (Email: kindhira@vit.ac.in) 
Theorem.3.1.3 Let IRAT $A_{\tilde{\alpha}}^{T}$ of A be a IRID of K for $\tilde{\alpha} \varepsilon$ $[0, \mathrm{I}]$, then the ensuing is valid,

i) If $\mathrm{x} \leq \square,\left(\lambda_{\mathrm{A}}\right)_{\tilde{\alpha}}^{\mathrm{T}}(\mathrm{x}) \geq\left(\lambda_{\mathrm{A}}\right)_{\tilde{\alpha}}^{\mathrm{T}}(\square) \&\left(\xi_{\mathrm{A}}\right)_{\tilde{\alpha}}^{\mathrm{T}}(\mathrm{x}) \leq\left(\xi_{\mathrm{A}}\right)_{\tilde{\alpha}}^{\mathrm{T}}(\square)$.

Proof. Let $\mathrm{x}, \square \varepsilon \dot{\mathrm{K}}, \mathrm{x} \leq \square, \mathrm{x} \diamond \square=0$, and this

$$
\begin{aligned}
\left(\lambda_{\mathrm{A}}\right)_{\tilde{\alpha}}^{\mathrm{T}}(\mathrm{x}) & \geq \min \left\{\left(\lambda_{\mathrm{A}}\right)_{\tilde{\alpha}}^{\mathrm{T}}(\mathrm{x} \diamond \square),\left(\lambda_{\mathrm{A}}\right)_{\tilde{\alpha}}^{\mathrm{T}}(\square)\right\} \\
& =\min \left\{\left(\lambda_{\mathrm{A}}\right)_{\tilde{\alpha}}^{\mathrm{T}}(0),\left(\lambda_{\mathrm{A}}\right)_{\tilde{\alpha}}^{\mathrm{T}}(\mathrm{a})\right\} \\
\left(\lambda_{\mathrm{A}}\right)_{\tilde{\alpha}}^{\mathrm{T}}(\mathrm{x}) & \geq\left(\lambda_{\mathrm{A}}\right)_{\tilde{\alpha}}^{\mathrm{T}}(\mathrm{a})
\end{aligned}
$$

and

$$
\begin{aligned}
&\left(\xi_{A}\right)_{\tilde{\alpha}}^{\mathrm{T}}(\mathrm{x}) \leq \max \left\{\left(\xi_{\mathrm{A}}\right)_{\tilde{\tilde{T}}}^{\mathrm{T}}(\mathrm{x} \diamond \square),\left(\xi_{\mathrm{A}}\right)_{\tilde{\alpha}}^{\mathrm{T}}(\square)\right\} \\
&=\max \left\{\left(\xi_{\mathrm{A}}\right)_{\tilde{\alpha}}^{\mathrm{T}}(0),\left(\xi_{\mathrm{A}}\right)_{\tilde{\alpha}}^{\mathrm{T}}(\mathrm{a})\right\} \\
&\left(\xi_{\mathrm{A}}\right)_{\tilde{\alpha}}^{\mathrm{T}}(\mathrm{x}) \leq\left(\xi_{\mathrm{A}}\right)_{\tilde{\alpha}}^{\mathrm{T}}(\mathrm{a}) .
\end{aligned}
$$

Theorem.3.1.4 If Let $A=\left(\lambda_{A}, \xi_{A}\right)$ is an IF_Ss of Ḱ, such that the IF_ $\tilde{\alpha}-$ translation $A_{\tilde{\alpha}}^{\mathrm{T}}=\left(\left(\lambda_{\mathrm{A}}\right)_{\tilde{\alpha}}^{\mathrm{T}},\left(\xi_{\mathrm{A}}\right)_{\tilde{\alpha}}^{\mathrm{T}}\right)$ of $\mathrm{A}$ is an IFINK_I of $\dot{\mathrm{K}}$ for $\tilde{\alpha} \varepsilon[0, \mathrm{I}]$, then $\tau_{\lambda_{\mathrm{A}}}=\{\mathrm{x} / \mathrm{x} \varepsilon \dot{\mathrm{K}}$ and $\left.\left(\lambda_{A}\right)_{\tilde{\alpha}}^{\mathrm{T}}(\mathrm{x})=\left(\lambda_{\mathrm{A}}\right)_{\tilde{\alpha}}^{\mathrm{T}}(0)\right\} \& \tau_{\xi_{A}}=\left\{\mathrm{x} / \mathrm{x} \varepsilon \dot{K}\right.$ and $\left(\xi_{\mathrm{A}}\right)_{\tilde{\alpha}}^{\mathrm{T}}(\mathrm{x})=$ $\left.\left(\xi_{\mathrm{A}}\right)_{\tilde{\alpha}}^{\mathrm{T}}(0)\right\}$ are INK_I of $\mathrm{K}$.

Proof. $A_{\tilde{\alpha}}^{\mathrm{T}}=\left(\left(\lambda_{A}\right)_{\tilde{\alpha}}^{\mathrm{T}},\left(\xi_{A}\right)_{\tilde{\alpha}}^{\mathrm{T}}\right)$ of $\mathrm{A}$ is an $\mathrm{IF}$ - ideal of $\mathrm{X}$. Then, $\left(\lambda_{A}\right)_{\tilde{\alpha}}^{\mathrm{T}}$ and $\left(\xi_{A}\right)_{\tilde{\alpha}}^{\mathrm{T}}$ are IFINK_I of Ḱ. Clearly

0 in $\tau_{\lambda \mathrm{A}}, \tau_{\Xi \mathrm{a}}$

Let $\mathrm{x}, \square, \mathrm{z} \varepsilon \dot{\mathrm{K}}$ be $((\mathrm{z} \diamond \mathrm{x}) \diamond(\mathrm{z} \diamond \square)) \varepsilon \tau_{\lambda_{\mathrm{A}}} \square \varepsilon \tau_{\lambda_{\mathrm{A}}}$,

$$
\begin{aligned}
\left(\lambda_{\mathrm{A}}\right)_{\tilde{\alpha}}^{\mathrm{T}}(\mathrm{z} \diamond \mathrm{x}) \diamond(\mathrm{z} \diamond \square) & =\left(\lambda_{\mathrm{A}}\right)_{\tilde{\alpha}}^{\mathrm{T}}(0) \\
\left(\lambda_{\mathrm{A}}\right)_{\tilde{\alpha}}^{\mathrm{T}}(\square) & =\left(\lambda_{\mathrm{A}}\right)_{\tilde{\alpha}}^{\mathrm{T}}(0)
\end{aligned}
$$

$\left(\lambda_{\mathrm{A}}\right)_{\tilde{\alpha}}^{\mathrm{T}}(\mathrm{x}) \geq \min \left\{\left(\lambda_{\mathrm{A}}\right)_{\tilde{\alpha}}^{\mathrm{T}}(\mathrm{z} \diamond \mathrm{x}) \diamond(\mathrm{z} \diamond \square),\left(\lambda_{\mathrm{A}}\right)_{\tilde{\alpha}}^{\mathrm{T}}(\square)\right\}$

$\left(\lambda_{\mathrm{A}}\right)_{\tilde{\alpha}}^{\mathrm{T}}(\mathrm{x})=\left(\lambda_{\mathrm{A}}\right)_{\tilde{\alpha}}^{\mathrm{T}}(0)$

$$
=\min \left\{\left(\lambda_{A}\right)_{\tilde{\alpha}}^{\mathrm{T}}(0),\left(\lambda_{\mathrm{A}}\right)_{\tilde{\alpha}}^{\mathrm{T}}(0)\right\}
$$

Since, $\left(\lambda_{A}\right)_{\tilde{\alpha}}^{\mathrm{T}}$ is a fuzzy INK-ideal

This implies

$$
\begin{aligned}
& \left(\lambda_{A}\right)_{\tilde{\alpha}}^{\mathrm{T}}(0) \geq\left(\lambda_{A}\right)_{\tilde{\alpha}}^{\mathrm{T}}(\mathrm{x}) \\
& \left(\lambda_{\mathrm{A}}\right)_{\tilde{\alpha}}^{\mathrm{T}}(\mathrm{x})=\left\{\left(\lambda_{\mathrm{A}}\right)_{\tilde{\alpha}}^{\mathrm{T}}(0)\right.
\end{aligned}
$$

$$
\lambda_{\mathrm{A}}(\mathrm{x})+\tilde{\alpha}=\lambda_{\mathrm{A}}(0)+\tilde{\alpha}(\text { or })
$$

$\lambda_{A}(x)=\lambda_{A}(0)$. So $x \varepsilon \tau_{\lambda_{A}}$ therefore $\tau_{\lambda_{A}}$ is an INK-ideal.

Yet again, $(\mathrm{a} \diamond \mathrm{b}) \diamond \mathrm{c} \varepsilon \tau_{\xi \mathrm{A}} \& \mathrm{~b} \varepsilon \tau_{\xi}$,

$$
\left(\xi_{A}\right)_{\tilde{\alpha}}^{\mathrm{T}}(c \diamond a) \diamond(c \diamond b)=\left(\xi_{A}\right)_{\tilde{\alpha}}^{\mathrm{T}}(0) \text { and, }
$$

$$
\left(\xi_{\mathrm{A}}\right)_{\tilde{\alpha}}^{\mathrm{T}}(\mathrm{b})=\left(\xi_{\mathrm{A}}\right)_{\tilde{\alpha}}^{\mathrm{T}}(0) \&
$$

$\left(\xi_{A}\right)_{\tilde{\alpha}}^{\mathrm{T}}(\mathrm{a}) \leq \max \left\{\left(\xi_{\mathrm{A}}\right)_{\tilde{\alpha}}^{\mathrm{T}}(\mathrm{c} \diamond \mathrm{a}) \diamond(\mathrm{c} \diamond \mathrm{b}),\left(\mathrm{v}_{\mathrm{A}}\right)_{\tilde{\alpha}}^{\mathrm{T}}(\mathrm{b})\right\}$

$$
\begin{gathered}
=\max _{\left.\left(\xi_{A}\right)_{\tilde{\alpha}}^{\mathrm{T}}\left(\xi_{A}\right)_{\tilde{\alpha}}^{\mathrm{T}}(0),\left(\xi_{A}\right)_{\tilde{\alpha}}^{\mathrm{T}}(0)\right\}}=\left(\xi_{A}\right)_{\tilde{\alpha}}^{\mathrm{T}}(0)
\end{gathered}
$$

$\left(\xi_{\mathrm{A}}\right)_{\tilde{\alpha}}^{\mathrm{T}}$ is a $\mathrm{F}_{-} \mathrm{INK}_{-} \mathrm{I}$

$$
\begin{aligned}
& \left(\xi_{A}\right)_{\tilde{\alpha}}^{\mathrm{T}}(0) \leq\left(\xi_{A}\right)_{\tilde{\alpha}}^{\mathrm{T}}(\mathrm{x}) \\
& \left(\xi_{A}\right)_{\tilde{\alpha}}^{\mathrm{T}}(\mathrm{x})=\left\{\left(\xi_{A}\right)_{\tilde{\alpha}}^{\mathrm{T}}(0)\right.
\end{aligned}
$$

This implies $\xi_{A}$ (a) $-\tilde{\alpha}=\xi_{A}(0)-\tilde{\alpha}$ (or)

$\xi_{A}(a)=\xi_{A}(0)$ so that a $\varepsilon \tau_{\xi A}$ therefore $\tau_{\lambda_{A}}$ is an INK-ideal.

Theorem.3.1.5 Let the IF_ $\tilde{\alpha}$-translation $A_{\tilde{\alpha}}^{\mathrm{T}}=\left(\left(\lambda_{A}\right)_{\tilde{\alpha}}^{\mathrm{T}}\right.$, $\left.\left(\xi_{A}\right)_{\tilde{\alpha}}^{\mathrm{T}}\right)$ of $\mathrm{A}$ is an IFINK-ideal of $\mathrm{K}$, for all $\tilde{\alpha}$ in $[0,1]$,

If $\mathrm{x} \geq \square,\left(\lambda_{\mathrm{A}}\right)_{\tilde{\alpha}}^{\mathrm{T}}\left(\mathrm{x}_{\mathrm{A}}\right) \geq\left(\lambda_{\mathrm{A}}\right)_{\tilde{\alpha}}^{\mathrm{T}}(\square) \&\left(\xi_{\mathrm{A}}\right)_{\tilde{\alpha}}^{\mathrm{T}}\left(\mathrm{x}_{\mathrm{A}}\right) \leq$ $\left(\xi_{\mathrm{A}}\right)_{\tilde{\alpha}}^{\mathrm{T}}(\square)$.

Proof. Let $\mathrm{x}, \square \varepsilon \dot{\mathrm{K}} . . \mathrm{x} \leq \square, \mathrm{x} \diamond \square=0$

we have,

$$
\begin{aligned}
&\left(\lambda_{\mathrm{A}}\right)_{\tilde{\alpha}}^{\mathrm{T}}(\mathrm{x}) \geq \min \left\{\left(\lambda_{\mathrm{A}}\right)_{\tilde{\alpha}}^{\mathrm{T}}(\mathrm{z} \diamond \mathrm{x}) \diamond(\mathrm{z} \diamond \square),\left(\lambda_{\mathrm{A}}\right)_{\tilde{\alpha}}^{\mathrm{T}}(0)\right\} \\
&= \min \left\{\left(\lambda_{\mathrm{A}}\right)_{\tilde{\alpha}}^{\mathrm{T}}(\mathrm{x} \diamond \square),\left(\lambda_{\mathrm{A}}\right)_{\tilde{\alpha}}^{\mathrm{T}}(\square)\right\} \\
&= \min \left\{\left(\lambda_{\mathrm{A}}\right)_{\tilde{\alpha}}^{\mathrm{T}}(0),\left(\lambda_{\mathrm{A}}\right)_{\tilde{\alpha}}^{\mathrm{T}}(\square)\right\} \\
&\left(\lambda_{\mathrm{A}}\right)_{\tilde{\alpha}}^{\mathrm{T}}(\mathrm{x})=\left(\lambda_{\mathrm{A}}\right)_{\tilde{\alpha}}^{\mathrm{T}}(\square)
\end{aligned}
$$

And,

$$
\begin{aligned}
\left(\xi_{A}\right)_{\tilde{\alpha}}^{T}(\mathrm{x}) & \leq \max \left\{\left(\xi_{A}\right)_{\tilde{\alpha}}^{T}(\mathrm{Z} \diamond \mathrm{x}) \diamond(\mathrm{Z} \diamond \square),\left(\xi_{A}\right)_{\tilde{\alpha}}^{T}(0)\right\} \\
& =\max \left\{\left(\xi_{A}\right)_{\tilde{\alpha}}^{T}(\mathrm{x} \diamond \square),\left(\xi_{A}\right)_{\tilde{\alpha}}^{T}(\square)\right\} \\
& =\max \left\{\left(\xi_{A}\right)_{\tilde{\alpha}}^{T}(0),\left(\xi_{A}\right)_{\tilde{\alpha}}^{T}(\square)\right\} \\
\left(\xi_{A}\right)_{\tilde{\alpha}}^{T}(\mathrm{x}) & =\left(\xi_{A}\right)_{\tilde{\alpha}}^{T}(\square) .
\end{aligned}
$$

\subsection{PROPERTIES ON IFINK-IDEAL EXTENSION.}

Definition.3.2.1 Let $A=\left(\lambda_{A}, \xi_{A}\right)$ and $B=\left(\lambda_{B}, \xi_{B}\right)$ be two IF_ sub set of K.. If $A \leq B . \lambda_{A}\left(x_{1}\right) \leq \lambda_{B}\left(x_{1}\right), \xi_{A}\left(x_{1}\right) \geq$ $\xi_{B}\left(x_{1}\right)$ for all $x_{\varepsilon} \varepsilon$ K, then $B$ is $I_{-}$extension of $A$.

Definition.3.3.2 Let $A=\left(\lambda_{A}, \xi_{A}\right)$ and $B=\left(\lambda_{B}, \xi_{B}\right)$ be two IF_subsets of $K$. Then $B$ is an IFINK_IE of $A$ is the ensuing statements are effective.

i) $\mathrm{B}$ is an $\mathrm{IF}_{-} \mathrm{E}$ of $\mathrm{A}$

ii) If $\mathrm{A}$ is an IFINK- I of Ḱ, the B is an IFINK-I of Ḱ.

From the definition of $\mathrm{IF}_{-} \tilde{\alpha}$-translation, $\left(\lambda_{A}\right)_{\tilde{\alpha}}^{\mathrm{T}}(\mathrm{x})$ $=\lambda_{A}\left(x_{1}\right)+\tilde{\alpha}$ and $\left(\xi_{A}\right)_{\tilde{\alpha}}^{T}\left(x^{2}\right)=\xi_{A}\left(x_{1}\right)-\tilde{\alpha}$ for each $x_{\varepsilon} \varepsilon \dot{K}$.

Theorem.3.3.3 Let $A=\left(\lambda_{A}, \xi_{A}\right)$ be an IFINK-ideal of Ḱ. and $\tilde{\alpha}$ in $[0, \tau]$. Then the $\mathrm{IF}_{-} \tilde{\alpha}_{-}$translation $\mathrm{A}_{\tilde{\alpha}}^{\mathrm{T}}=\left(\left(\lambda_{\mathrm{A}}\right)_{\tilde{\alpha}}^{\mathrm{T}}\right.$, $\left.\left(\xi_{A}\right)_{\tilde{\alpha}}^{\mathrm{T}}\right)$ of A is an IFINK-ideal of A. An IFINK-ideal A ma not be denoted as an $\mathrm{IF}_{-} \tilde{\alpha}_{-}$translation of $\mathrm{A}$, that is the reverse of Theorem is not correct in universal as understood in the ensuing example.

Example.3.3.4 Let Ḱ. $=\{0, a, b, 1,2\}$ be a INK-algebra per the ensuing Cayley table

\begin{tabular}{|c|c|c|c|c|c|}
\hline$\circ$ & 0 & $\mathrm{a}$ & $\mathrm{b}$ & 1 & 2 \\
\hline 0 & 0 & 0 & 0 & 0 & 0 \\
\hline $\mathrm{a}$ & $\mathrm{a}$ & 0 & $\mathrm{a}$ & 0 & 0 \\
\hline $\mathrm{b}$ & $\mathrm{b}$ & 2 & 0 & 0 & 0 \\
\hline 1 & 1 & 1 & 1 & 0 & 0 \\
\hline 2 & 2 & 1 & 2 & $\mathrm{a}$ & 0 \\
\hline
\end{tabular}

Let $A=\left(\lambda_{A}, \xi_{A}\right)$ be an IFSs of $K$, clearly by

\begin{tabular}{|c|ccccc|}
\hline$\dot{\mathrm{K}}$ & 0 & $\mathrm{a}$ & $\mathrm{b}$ & 1 & 2 \\
\hline$\mu_{A}$ & 0.62 & 0.54 & 0.45 & 0.31 & 0.31 \\
\hline$\xi_{A}$ & 0.17 & 0.22 & 0.30 & 0.45 & 0.45 \\
\hline
\end{tabular}

Then $A=\left(\lambda_{A}, \xi_{A}\right)$ be an IFINK-ideal of Ḱ.

Let $\mathrm{B}=\left(\lambda_{\mathrm{B}}, \xi_{\mathrm{B}}\right)$ be an $\mathrm{IF}_{-}$subset of $\mathrm{K}$, defined $\mathrm{b}$

\begin{tabular}{|l|ccccc|}
\hline$\dot{\mathrm{K}}$ & 0 & $\mathrm{a}$ & $\mathrm{b}$ & 1 & 2 \\
\hline$\lambda_{B}$ & 0.64 & 0.60 & 0.48 & 0.35 & 0.35 \\
\hline$\xi_{B}$ & 0.15 & 0.18 & 0.27 & 0.41 & 0.41 \\
\hline
\end{tabular}

$A=\left(\lambda_{A}, \xi_{A}\right)$ be an IFINK_I extension of A. But it is not the IF_ $\tilde{\alpha}$-Translation $A_{\tilde{\alpha}}^{\mathrm{T}}=\left(\left(\lambda_{A}\right)_{\tilde{\alpha}}^{\mathrm{T}},\left(\xi_{A}\right)_{\tilde{\alpha}}^{\mathrm{T}}\right)$ of A for all $\tilde{\alpha}$ in $[0$, $\tau]$. If $\tilde{\alpha}=0.16, \tilde{\alpha}=0.16>0.13=\lambda$. And the IF_ $\tilde{\alpha}$-translation $\left.A_{\tilde{\alpha}}^{\mathrm{T}}=\left(\lambda_{\mathrm{A}}\right)_{\tilde{\alpha}}^{\mathrm{T}},\left(\xi_{\mathrm{A}}\right)_{\tilde{\alpha}}^{\mathrm{T}}\right)$ of $\mathrm{A}$ is assumed as follows

\begin{tabular}{|c|ccccc|}
\hline$\dot{K}$ & 0 & $\mathrm{a}$ & $\mathrm{b}$ & 1 & 2 \\
\hline$\left(\lambda_{\mathrm{A}}\right)_{\tilde{\alpha}}^{\mathrm{T}}$ & 0.71 & 0.58 & 0.50 & 0.58 & 0.50 \\
\hline$\left(\xi_{\mathrm{A}}\right)_{\tilde{\alpha}}^{\mathrm{T}}$ & 0.27 & 0.41 & 0.52 & 0.41 & 0.52 \\
\hline
\end{tabular}

Therefore, $\left(\lambda_{A}\right)_{\tilde{\alpha}}^{\mathrm{T}}+\left(\xi_{\mathrm{A}}\right)_{\tilde{\alpha}}^{\mathrm{T}} \neq 1$. Therefore, B is not the IF

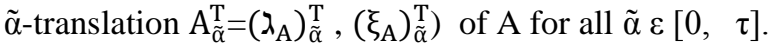


Theorem.3.3.5 Let $A=\left(\lambda_{A}, \xi_{A}\right)$ be an IFINK-I of $K$ and $\tilde{\alpha}$ in $[0, \tau]$. Then the IF_ $\tilde{\alpha}$-translation $A_{\tilde{\alpha}}^{\mathrm{T}}=\left(\left(\lambda_{\mathrm{A}}\right)_{\tilde{\alpha}}^{\mathrm{T}},\left(\xi_{\mathrm{A}}\right)_{\tilde{\alpha}}^{\mathrm{T}}\right)$ of $\mathrm{A}$ is an IFINK_I extension of A. Then intersection of IFINK I of $\mathrm{K}$ is an IFINK_ I extension of A. But the union of IFI NK_ I extensions of an IFINK_I extension of as understood in the ensuing.

Example.3.3.6 Let $A=\left(\lambda_{A}, \xi_{A}\right)$ be an IFINK-ideal of $K$ and $\tilde{\alpha} \varepsilon[0, \tau]$. Then the IF_ $\tilde{\alpha}$-translation $\left.A_{\tilde{\alpha}}^{\mathrm{T}}=\left(\lambda_{A}\right)_{\tilde{\alpha}}^{\mathrm{T}},\left(\xi_{A}\right)_{\tilde{\alpha}}^{\mathrm{T}}\right)$ of $\mathrm{A}$ is an IFINK-ideal extension of $\mathrm{K}$. Then intersection of IFINK-ideal extension of an IF_INK-ideal A of Ḱ is an IFI NK- ideal extension of A. But the union of IFINK-extensions of an IF_INK-A of $K$ is not an IFINK-extension of a as seen in the following example.

Example.3.3.7 Let $\mathrm{K}=\{0,1,2,3,4\}$ be a INK-algebra by means of the succeeding Cayley table.

\begin{tabular}{|c|c|c|c|c|c|}
\hline 0 & 0 & $\mathrm{a}$ & $\mathrm{b}$ & 1 & 2 \\
\hline 0 & 0 & 0 & 0 & 0 & 0 \\
\hline $\mathrm{a}$ & $\mathrm{a}$ & 0 & $\mathrm{a}$ & 0 & 0 \\
\hline $\mathrm{b}$ & $\mathrm{b}$ & 2 & 0 & 0 & $\mathrm{~b}$ \\
\hline 1 & 1 & 2 & $\mathrm{a}$ & 0 & $\mathrm{~b}$ \\
\hline 2 & 2 & $\mathrm{a}$ & 2 & $\mathrm{a}$ & 0 \\
\hline
\end{tabular}

Let $A=\left(\lambda_{A}, \xi_{A}\right)$ be an IF_Ss of Ḱ, well-defined by

\begin{tabular}{|l|lllll|}
\hline$\dot{\mathrm{K}}$ & 0 & $\mathrm{a}$ & $\mathrm{b}$ & 1 & 2 \\
\hline$\lambda_{A}$ & 0.53 & 0.31 & 0.31 & 0.31 & 0.31 \\
\hline$\xi_{A}$ & 0.25 & 0.48 & 0.48 & 0.48 & 0.48 \\
\hline
\end{tabular}

Before $A=\left(\lambda_{A}, \xi_{A}\right)$ be an IFINK_I of K

Let $\mathrm{B}=\left(\lambda_{\mathrm{B}}, \xi_{\mathrm{B}}\right)$ and $\mathrm{C}=\left(\lambda_{\mathrm{C}}, \xi_{\mathrm{C}}\right)$ be an $\mathrm{IF}_{-}$Ss of $\mathrm{K}$ correspondingly by

\begin{tabular}{|l|rrrrrr|}
\hline K & 0 & a & b & 1 & 2 & \\
\hline$\lambda_{B}$ & & 0.66 & 0.56 & 0.33 & 0.33 & 0.56 \\
\hline$\xi_{B}$ & & 0.12 & 0.23 & 0.44 & 0.44 & 0.23 \\
\hline
\end{tabular}

And

\begin{tabular}{|l|lllll|}
\hline$\dot{\mathrm{K}}$ & 0 & $\mathrm{a}$ & $\mathrm{b}$ & 1 & 2 \\
\hline$\lambda_{C}$ & 0.68 & 0.61 & 0.47 & 0.41 & 0.41 \\
\hline$\xi_{C}$ & 0.11 & 0.35 & 0.31 & 0.35 & 0.35 \\
\hline
\end{tabular}

Then $\mathrm{B} \& \mathrm{C}$ are IFINK-I of A

1) The intersection $B \cap C$ is an IF_extension of $A$, $\lambda_{\mathrm{B} \cap \mathrm{C}}(1) \geq \min \left\{\lambda_{\mathrm{B} \cap \mathrm{C}}(0 \diamond 1) \diamond(0 \diamond 1), \lambda_{\mathrm{B} \cap \mathrm{C}}(1)\right\}$ $\min \left\{\lambda_{\mathrm{B}}(1), \lambda_{\mathrm{C}}(1)\right\} \geq \min \left\{\lambda_{\mathrm{B} \cap \mathrm{C}}(0), \lambda_{\mathrm{B} \cap \mathrm{C}}(0)\right\}$ $\min \{0.33,0.41\} \geq \min \{0.66,0.33\}$

$$
0.33=0.33
$$

$$
\xi_{\mathrm{B} \cap \mathrm{C}}(1) \leq \max \left\{\xi_{\mathrm{B} \cap \mathrm{C}}(0 \diamond 1) \diamond(0 \diamond \mathrm{b}), \xi_{\mathrm{B} \cap \mathrm{C}}(\mathrm{b})\right\}
$$

$\max \{0.44,0.35\} \leq \max \left\{\xi_{\mathrm{B} \cap \mathrm{C}}(0), \xi_{\mathrm{B} \cap \mathrm{C}}(\mathrm{b})\right\}$ $\max \{0.44,0.35\} \leq \max \{0.12,0.44\}$

$$
0.44=0.44
$$

2) The union $B U C$ is an $I F_{-} E$ of $A$, it is not an $I F_{-} E$ of $A$ $\lambda_{\text {BUC }}(1) \geq \min \left\{\lambda_{\text {BUC }}(0 \diamond 1) \diamond(0 \diamond b), \lambda_{\text {BUC }}(b)\right\}$ $\min \{0.33,0.31\} \geq \min \left\{\lambda_{\mathrm{BUC}}(0), \lambda_{\mathrm{BUC}}(\mathrm{b})\right\}$

\author{
$\min \{0.41\} \geq \min \{0.68,0.47\}$ \\ $0.41 \geq 0.47$ \\ $\left.\xi_{\mathrm{BUC}}(1) \leq \max \left\{\xi_{\mathrm{BUC}}(0 \diamond 1) \diamond(0 \diamond \mathrm{b}), \xi_{\mathrm{BUC}} \mathrm{b}\right)\right\}$ \\ $\max \{0.35,0.44\} \leq \max \left\{\xi_{\text {BUC }}(0), \xi_{\text {BUC }}(\right.$ b) $\}$ \\ $\max \{0.35\} \leq \max \{0.11,0.31\}$ \\ $0.35 \leq 0.31$
}

Theorem.3.3.8 If $\mathrm{A}$ is an IFINK-ideal of $\mathrm{K}$, formerly it is pure that $\mathrm{U}_{\tilde{\alpha}}\left(\lambda_{\tilde{\alpha}} ; \mathrm{t}\right)$ and $\mathrm{L}_{\tilde{\alpha}}\left(\xi_{\tilde{\alpha}} ; \mathrm{s}\right)$ are $\mathrm{INK}_{-} \mathrm{I}$ of $\mathrm{K}$ for all $\mathrm{t} \varepsilon \mathrm{I}_{\mathrm{m}}$ $\left(\lambda_{\mathrm{A}}\right) \& \mathrm{~s} \& \mathrm{I}_{\mathrm{m}}\left(\xi_{\mathrm{A}}\right)$ with $\mathrm{t} \geq \tilde{\alpha}$. But, if we do not give a condition that A is an IFINK_I of X, $\mathrm{U}_{\tilde{\alpha}}\left(\lambda_{\tilde{\alpha}} ; \mathrm{t}\right) \& \mathrm{~L}_{\tilde{\alpha}}\left(\xi_{\tilde{\alpha}} ; \mathrm{s}\right)$ are not INK-ideal of $K$ as perceived in the succeeding example.

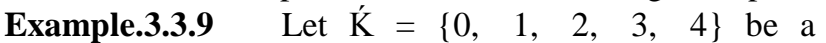
INK-algebra in example 2 and $A=\left(\lambda_{A}, \xi_{A}\right)$ be an IF_ Ss of K defined by

\begin{tabular}{|c|lllll|}
\hline$\dot{\mathrm{K}}$ & 0 & $\mathrm{a}$ & $\mathrm{b}$ & 1 & 2 \\
\hline$\lambda_{A}$ & 0.56 & 0.39 & 0.21 & 0.39 & 0.39 \\
\hline$\xi_{A}$ & 0.23 & 0.38 & 0.57 & 0.38 & 0.38 \\
\hline
\end{tabular}

$$
\begin{gathered}
\lambda_{\mathrm{A}}(1) \geq \min \left\{\lambda_{\mathrm{A}}(1 \diamond \mathrm{a}) \diamond(1 \diamond 0), \lambda_{\mathrm{A}}(0)\right\} \\
0.39 \geq \min \left\{\lambda_{\mathrm{A}}(\mathrm{b} \diamond 1), \lambda_{\mathrm{A}}(0)\right\} \\
0.39 \geq \min \left\{\lambda_{\mathrm{A}}(0), \lambda_{\mathrm{A}}(0)\right\} \\
0.39 \geq \min \{0.56,0.56\} \\
0.39 \geq 0.56
\end{gathered}
$$

$\xi_{A}(3) \geq \min \left\{\xi_{A}(0), \xi_{A}(0)\right\}$ $0.28 \geq \min \{0.13,0.13\}$

\section{$0.28 \leq / 0.13$}

Therefore, $A=\left(\lambda_{A}, \xi_{A}\right)$ is not an IFINK-I of Ḱ. For, $\tilde{\alpha}=$ $0.16, \mathrm{t}=0.60$ and $\mathrm{s}=0.45$. We acquire $\mathrm{U}_{\tilde{\alpha}}\left(\lambda_{\tilde{\alpha}} ; \mathrm{t}\right)=\mathrm{L}_{\tilde{\alpha}}\left(\xi_{\tilde{\alpha}} ; \mathrm{s}\right)$ $=\{0,1,2,3,4\}$ which are not INK -ideal of Ḱ. Level sets in IF_ $\tilde{\alpha}$-translations

Theorem.3.3.10 Let $A=\left(\lambda_{A}, \xi_{A}\right)$ be an $\mathrm{IF}_{-}$Ss of Ḱ \& $\tilde{\alpha} \varepsilon$ $[0, T]$, then the IF $-\tilde{\alpha}$-translation $A_{\tilde{\alpha}}^{\mathrm{T}}=\left(\lambda_{A}\right)_{\tilde{\alpha}}^{\mathrm{T}},\left(\xi_{A}\right)_{\tilde{\alpha}}^{\mathrm{T}}$ of $\mathrm{A}$ is an IFINK-I of $\mathrm{K}$ if and only if $\mathrm{U}_{\tilde{\alpha}}\left(\lambda_{\tilde{\alpha}} ; \mathrm{t}\right)$ and $\mathrm{L}_{\tilde{\alpha}}\left(\xi_{\tilde{\alpha}} ; \mathrm{s}\right)$ are I. NK-I of Ḱ, for $\mathrm{t} \varepsilon \mathrm{I}_{\mathrm{m}}\left(\lambda_{\mathrm{A}}\right)$ and $\mathrm{s} \varepsilon \mathrm{I}_{\mathrm{m}}\left(\xi_{\mathrm{A}}\right)$ with $\mathrm{t} \geq \tilde{\alpha}$.

Proof. Suppose that $A_{\tilde{\alpha}}^{T}=\left(\lambda_{A}\right)_{\tilde{\alpha}}^{T},\left(\xi_{A}\right)_{\tilde{\alpha}}^{T}$ of $A$ is an IFINK-ideal of Ḱ.

Then $\left(\lambda_{A}\right)_{\tilde{\alpha}}^{\mathrm{T}}$ and $\left(\xi_{\mathrm{A}}\right)_{\tilde{\alpha}}^{\mathrm{T}}$ are IF_ INK of Ḱ, let, $\mathrm{t} \varepsilon \mathrm{I}_{\mathrm{m}}\left(\lambda_{\mathrm{A}}\right)$ and $\mathrm{s} \& \mathrm{I}_{\mathrm{m}}\left(\xi_{\mathrm{A}}\right)$ with $\geq \tilde{\alpha}$.

$$
\begin{aligned}
& \text { Since, }\left(\lambda_{A}\right)_{\tilde{\alpha}}^{\mathrm{T}}(0) \geq\left(\lambda_{A}\right)_{\tilde{\alpha}}^{\mathrm{T}}\left(\mathrm{x}_{\mathrm{H}}\right) \\
& \lambda_{\mathrm{A}}(0)+\tilde{\alpha}=\left(\lambda_{\mathrm{A}}\right)_{\tilde{\alpha}}^{\mathrm{T}}(0) \\
& \geq\left(\lambda_{\mathrm{A}}\right)_{\tilde{\alpha}}^{\mathrm{T}}(\mathrm{x}) \\
& =\left(\lambda_{\mathrm{A}}\right)_{\tilde{\alpha}}^{\mathrm{T}}\left(\mathrm{x}_{\mathrm{A}}\right)+\tilde{\alpha} \\
& \geq \mathrm{t}, \mathrm{x} \varepsilon \mathrm{U}_{\tilde{\alpha}}\left(\lambda_{\tilde{\alpha}} ; \mathrm{t}\right) \\
& \sigma \varepsilon U_{\tilde{\alpha}}\left(\lambda_{\mathrm{A}} ; \mathrm{t}\right) \\
& \text { Let, } \mathrm{x}, \square, \mathrm{z} \varepsilon \mathrm{X},(\mathrm{z} \diamond \mathrm{x}) \diamond(\mathrm{z} \diamond \square), \square \varepsilon \mathrm{U}_{\tilde{\alpha}}\left(\lambda_{\mathrm{A}} ; \mathrm{t}\right) \\
& \lambda_{\mathrm{A}}((\mathrm{z} \diamond \mathrm{x}) \diamond(\mathrm{z} \diamond \square)) \geq \mathrm{t}-\tilde{\alpha} \& \lambda_{\mathrm{A}}(\square) \geq \mathrm{t}-\tilde{\alpha} \\
& \left(\lambda_{A}\right)_{\tilde{\alpha}}^{\mathrm{T}}((\mathrm{z} \diamond \mathrm{x}) \diamond(\mathrm{z} \diamond \square))=\lambda_{A}((\mathrm{z} \diamond \mathrm{x}) \diamond(\mathrm{z} \diamond \square))+\tilde{\alpha} \\
& \geq \mathrm{t} \\
& \left(\lambda_{A}\right)_{\tilde{\alpha}}^{\mathrm{T}}(\square)=\lambda_{A}(\square)+\tilde{\alpha} \\
& \lambda_{\mathrm{A}}(\mathrm{x})+\tilde{\alpha}=\left(\lambda_{\mathrm{A}}\right)_{\tilde{\alpha}}^{\mathrm{T}}\left(\mathrm{x}_{\mathrm{H}}\right) \\
& \geq \min \left\{\left(\lambda_{\mathrm{A}}\right)_{\tilde{\alpha}}^{\mathrm{T}}((\mathrm{z} \diamond \mathrm{x}) \diamond(\mathrm{z} \diamond \text { 目 })) \quad, \quad\left(\lambda_{\mathrm{A}}\right)_{\tilde{\alpha}}^{\mathrm{T}}(\square)\right\} \\
& =\min \{t, t\} \geq t \text {. }
\end{aligned}
$$




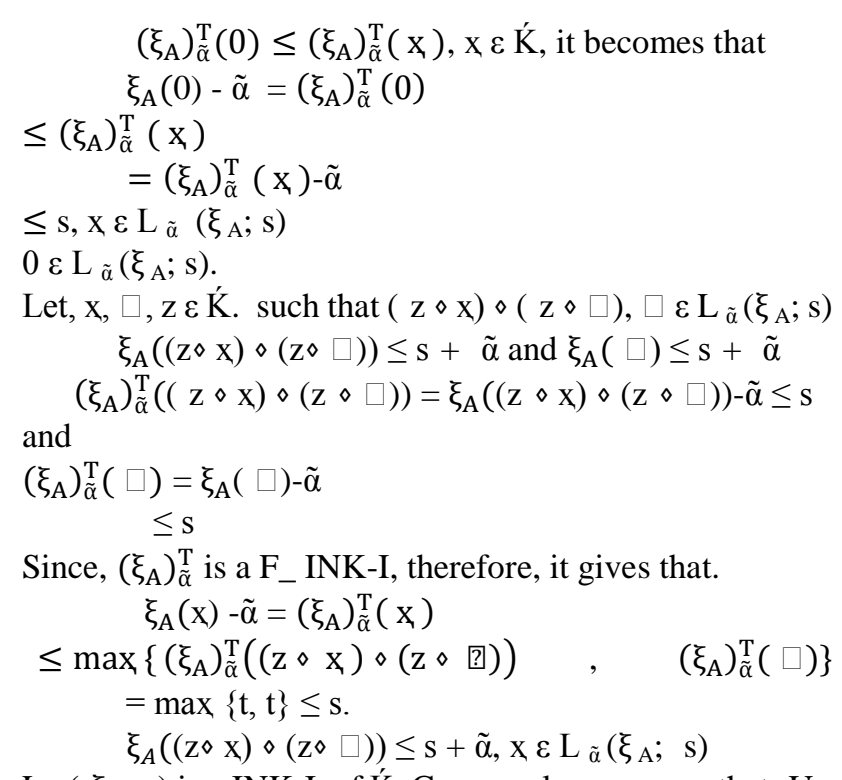

$\mathrm{L}_{\tilde{\alpha}}\left(\xi_{A} ; s\right)$ is a INK-I, of Ḱ. Conversely, suppose that $\mathrm{U}_{\tilde{\alpha}}$ $\left(\lambda_{A} ; \mathrm{t}\right) \& \mathrm{~L}_{\tilde{\alpha}}\left(\xi_{\mathrm{A}} ; \mathrm{s}\right)$ are INK- I of K for $\mathrm{t} \varepsilon \mathrm{I}_{\mathrm{m}}\left(\lambda_{\mathrm{A}}\right)$ and $\mathrm{s} \varepsilon \mathrm{I}_{\mathrm{m}}$ $\left(\xi_{\mathrm{A}}\right)$ with $\mathrm{t} \geq \tilde{\alpha}$. If them exist $\mathrm{U} \varepsilon \dot{\mathrm{K}}$, such that $\left(\lambda_{\mathrm{A}}\right)_{\tilde{\alpha}}^{\mathrm{T}}<\psi \leq$ $\left(\lambda_{A}\right)_{\tilde{\alpha}}^{\mathrm{T}}(\mathrm{u})$. Then, $\lambda_{A}(u) \geq \psi-\tilde{\alpha}$, but $\lambda_{A}(0)<\psi-\tilde{\alpha}$. This shows that $u \in U_{\tilde{\alpha}}\left(\lambda_{A} ; t\right) \& 0 E U_{\tilde{\alpha}}\left(\lambda_{A} ; t\right)$. This is contradiction, and $\left(\lambda_{A}\right)_{\tilde{\alpha}}^{T}(0) \geq\left(\lambda_{A}\right)_{\tilde{\alpha}}^{T}(x)$ for $x \varepsilon \dot{K}$. Over, if them exist $\mathrm{t} \xi$ in $K^{\prime}$, such that, $\left(\xi_{A}\right)_{\tilde{\alpha}}^{\mathrm{T}}(0)>X \geq\left(\xi_{A}\right)_{\tilde{\alpha}}^{\mathrm{T}}(\mathrm{v})$, then $\xi_{A}(v) \leq X+\tilde{\alpha}$, But, $\xi_{A}(\mathrm{v}) \geq \mathrm{X}+\tilde{\alpha}$. Show that $\xi \varepsilon \mathrm{L}_{\tilde{\alpha}}\left(\xi_{A} ; \mathrm{s}\right)$ and $0 \mathrm{EL}_{\tilde{\alpha}}\left(\xi_{\mathrm{A}} ; \mathrm{s}\right)$.

This is contradiction $\& \xi_{A}(0) \leq\left(\xi_{A}\right)_{\tilde{\alpha}}^{T}\left(x_{A}\right)$ for $\mathrm{x} \varepsilon \dot{K}$.

We undertake that there exists a, b, c $\varepsilon$ K, $\left(\lambda_{A}\right)_{\tilde{\alpha}}^{\mathrm{T}}(\mathrm{a}) \geq \mathrm{k}_{1} \leq$ $\min \left\{\left(\lambda_{\mathrm{A}}\right)_{\tilde{\alpha}}^{\mathrm{T}}(c \diamond \mathrm{a}) \diamond(c \diamond b),\left(\lambda_{\mathrm{A}}\right)_{\tilde{\alpha}}^{\mathrm{T}}(\mathrm{b})\right\}$. Then, $\left(\lambda_{\mathrm{A}}\right)_{\tilde{\alpha}}^{\mathrm{T}}(c \diamond \mathrm{a}) \diamond$ $(c \diamond b) \geq k_{1}-\tilde{\alpha}$ and $\lambda_{A}(b) \geq k_{1}-\tilde{\alpha}$. But, $\lambda_{A}(a)<k_{1}-\tilde{\alpha}$

Hence, $(c \diamond a) \diamond(c \diamond b) \varepsilon U_{\tilde{\alpha}}\left(\lambda_{A} ; t\right)$ and $b \varepsilon L_{\tilde{\alpha}}\left(\xi_{A} ; s\right)$.

But, a $E U_{\tilde{\alpha}}\left(\lambda_{\mathrm{A}} ; \mathrm{t}\right)$.

This stays contradiction.

$\left(\lambda_{\mathrm{A}}\right)_{\tilde{\alpha}}^{\mathrm{T}}(\mathrm{a}) \geq \min \left\{\left(\lambda_{\mathrm{A}}\right)_{\tilde{\alpha}}^{\mathrm{T}}(\mathrm{z} \diamond \mathrm{x}) \diamond(\mathrm{z} \diamond \theta),\left(\lambda_{\mathrm{A}}\right)_{\tilde{\alpha}}^{\mathrm{T}}(\square)\right\}$ for all $\mathrm{x}, \square, \mathrm{z} \varepsilon \dot{\mathrm{K}}$

Once more, adopt that there exist, $\mathrm{x}, \square, \mathrm{z}$ in $\mathrm{K}$

$\left(\lambda_{\mathrm{A}}\right)_{\tilde{\alpha}}^{\mathrm{T}}(\mathrm{a})<\eta \leq \max \left\{\left(\xi_{\mathrm{A}}\right)_{\tilde{\alpha}}^{\mathrm{T}}(\mathrm{z} \diamond \mathrm{x}) \diamond(\mathrm{z} \diamond\right.$ $\left.),\left(\xi_{\mathrm{A}}\right)_{\tilde{\alpha}}^{\mathrm{T}}(\square)\right\}$

Then,

$\left(\xi_{A}\right)_{\tilde{\alpha}}^{T}(x)<\eta+\tilde{\alpha}$ and $\xi_{A}(\square) \leq \eta+\tilde{\alpha}$

But, $\xi_{A}(\mathrm{v}) \geq \eta+\tilde{\alpha}$

Hence,

$(\mathrm{z} \diamond \mathrm{x}) \diamond\left(\mathrm{z} \diamond\right.$ ?) $\varepsilon \mathrm{L}_{\tilde{\alpha}}\left(\xi_{\mathrm{A}} ; \mathrm{s}\right)$ and $\square \varepsilon \mathrm{L}_{\tilde{\alpha}}\left(\xi_{\mathrm{A}} ; \mathrm{s}\right)$

But, $\mathrm{x} \mathrm{EL}_{\tilde{\alpha}}\left(\xi_{\mathrm{A}} ; \mathrm{s}\right)$, which is a contradiction.

Therefore,

$\left(\xi_{A}\right)_{\tilde{\alpha}}^{T}(x) \leq \min \left\{\left(\xi_{A}\right)_{\tilde{\alpha}}^{T}\left(\mathrm{z} \diamond \mathrm{x}_{1}\right) \diamond\left(\mathrm{z} \diamond\right.\right.$ ?), $\left.\left(\xi_{A}\right)_{\tilde{\alpha}}^{T}(y)\right\}$ for all $\mathrm{x}, \square, \mathrm{z} \varepsilon \dot{\mathrm{K}}$.

Thus $A_{\tilde{\alpha}}^{T}$ is an IFINK-ideals of Ḱ.

Definition.3.3.11 Let $A=\left(\lambda_{A}, \xi_{A}\right)$ be an IFS of $\dot{K}$ and Let e $\varepsilon[0,1]$. An object having the from $A_{e}^{M}=\left(\lambda_{A}\right)_{e}^{M},\left(\xi_{A}\right)_{e}^{M}$ is called an $\mathrm{IF}_{-}$e-multiplication of $\mathrm{A}$ if, $\left(\lambda_{\mathrm{A}}\right)_{\mathrm{e}}^{\mathrm{M}}(\mathrm{x})=e \cdot \mathrm{\lambda}_{\mathrm{A}}(\mathrm{x})$ and $\left(\xi_{A}\right)_{\mathrm{e}}^{M}(\mathrm{x})=$ e. $\xi_{A}(\mathrm{x})$, for all $\mathrm{x} \varepsilon \dot{\mathrm{K}}$. For an $\square \mathrm{IFS} \mathrm{A}=$ $\left(\lambda_{\mathrm{A}}, \xi_{\mathrm{A}}\right)$ of $\dot{\mathrm{K}}$, an IR 0-multiplication $A_{0}^{M}=\left(\mu_{A}\right)_{0}^{M},\left(\xi_{A}\right)_{0}^{M}$ of $\mathrm{A}$ is an INK-I of $\mathrm{K}$,

for all e $\varepsilon[0,1]$.

Theorem.3.3.12. Let $A=\left(\lambda_{A}, \xi_{A}\right)$ be an IF_ Ss of Ḱ such that IR e-multiplication of $A_{\mathrm{e}}^{M}$ of $\mathrm{K}$ is an IFINK-ideal of $\mathrm{A}$, for some e $\varepsilon[0,1]$, then $\mathrm{A}$ is an INK-ideal of Ḱ.

Proof. $\left(\mu_{A}\right)_{\mathrm{e}}^{M} \&\left(\xi_{A}\right)_{\mathrm{e}}^{M}$ is a IF_ INK of Ḱ for some e $\varepsilon$ $[0,1]$. Let $\mathrm{x}, \square, \mathrm{z} \varepsilon \dot{\mathrm{K}}$.

$$
\begin{aligned}
& \quad \text { e. } \lambda_{A}(0) \geq \\
& \geq\left(\lambda_{A}\right)_{e}^{\mathrm{M}}(\mathrm{x}) \\
& \text { e. } \lambda_{A}(0)=\lambda_{A}\left(x_{A}\right)_{\mathrm{e}}^{\mathrm{M}} \\
& \text { e. e } \xi_{\mathrm{A}}(0) \leq \\
& \leq\left(\xi_{\mathrm{A}}\right)_{\mathrm{e}}^{\mathrm{M}}(\mathrm{x}) \\
& \text { e. } \xi_{\mathrm{A}}(0)=\xi_{\mathrm{A}}(\mathrm{x}) \cdot \mathrm{e} \\
& \text { so, } \\
& \lambda_{A}(0) \geq \lambda_{A}(\mathrm{x}) \\
& \xi_{\mathrm{A}}(0) \leq \xi_{\mathrm{A}}(\mathrm{x})
\end{aligned}
$$

Also,

e. $\lambda_{\mathrm{A}}(\mathrm{x})=\left(\lambda_{\mathrm{A}}\right)_{\mathrm{e}}^{\mathrm{M}}(\mathrm{x})$ $\geq \min \left\{\left(\lambda_{\mathrm{A}}\right)_{\mathrm{e}}^{\mathrm{M}}(\mathrm{z} \diamond \mathrm{x}) \diamond(\mathrm{z} \diamond\right.$ $\left.),\left(\lambda_{\mathrm{A}}\right)_{\mathrm{e}}^{\mathrm{M}}(\square)\right\}$

$\geq \min \left\{\right.$ e. $\lambda_{A}(z \diamond x) \diamond(z \diamond$ ? $)$, e. $\lambda_{A}($ 圆 $\left.)\right\}$

e. $\lambda_{A}(x) \geq e \cdot \min \left\{\lambda_{A}(z \diamond x) \diamond(z \diamond\right.$ ? $\left.), \lambda_{A}(?)\right\}$

and,

$$
\text { e. } \xi_{\mathrm{A}}(\mathrm{x})=\left(\xi_{\mathrm{A}}\right)_{\mathrm{e}}^{\mathrm{M}}
$$

$\leq \max \left\{\left(\xi_{\mathrm{A}}\right)_{\mathrm{e}}^{\mathrm{M}}(\mathrm{z} \diamond \mathrm{x}) \diamond(\mathrm{z} \diamond \vartheta),\left(\xi_{\mathrm{A}}\right)_{\mathrm{e}}^{\mathrm{M}}(\square)\right\}$

$\leq \max \left\{\mathrm{e} \cdot \xi_{\mathrm{A}}(\mathrm{z} \diamond \mathrm{x}) \diamond(\mathrm{z} \diamond\right.$ ?) $), \mathrm{e} \cdot \xi_{\mathrm{A}}($ 目 $\left.)\right\}$

e. $\xi_{A}(x) \leq e \cdot \max \left\{\xi_{A}(z \diamond x) \diamond(z \diamond\right.$ ? $), \xi_{A}($ ? $\left.)\right\}$ and so,

$$
\begin{aligned}
& \lambda_{\mathrm{A}}(\mathrm{x}) \geq \min \left\{\lambda_{\mathrm{A}}(\mathrm{z} \diamond \mathrm{x}) \diamond(\mathrm{z} \diamond ?), \lambda_{\mathrm{A}}(\text { ? })\right\} \\
& \xi_{\mathrm{A}}(\mathrm{x}) \leq \max \left\{\xi_{\mathrm{A}}(\mathrm{z} \diamond \mathrm{x}) \diamond(\mathrm{z} \diamond ?), \xi_{\mathrm{A}}(\text { ? })\right\}
\end{aligned}
$$

Hence $A=\left(\lambda_{A}, \xi_{A}\right)$ be an IFINK-ideal of $K$

Theorem.3.3.13 If $A=\left(\lambda_{A}, \xi_{A}\right)$ is an IR_INK_I of $K$ then the INT-e-multiplication $A_{e}^{\mathrm{M}}=\left(\lambda_{\mathrm{A}}\right)_{\mathrm{e}}^{\mathrm{M}},\left(\xi_{\mathrm{A}}\right)_{\mathrm{e}}^{\mathrm{M}}$ of $\mathrm{A}$ is an INT- IF_INK of Ḱ, for some e $\varepsilon[0,1]$

Proof. Let $=\left(\lambda_{A}, \xi_{A}\right)$ is an IR_INK-I of Ḱ and let e $\varepsilon[0,1]$

Then,

$$
\begin{gathered}
\left(\lambda_{A}\right)_{e}^{\mathrm{M}}(0)=\text { e. } \lambda_{A}(0) \\
\geq \text { e. } \lambda_{A}(x) \\
\left(\lambda_{A}\right)_{e}^{M}=\left(\lambda_{A}\right)_{e}^{M}(x)
\end{gathered}
$$

And,

$$
\begin{aligned}
& \left(\lambda_{\mathrm{A}}\right)_{\mathrm{e}}^{\mathrm{M}}(\mathrm{x})=\text { e. } \lambda_{\mathrm{A}}(\mathrm{x}) \\
& \geq \text { e. } \min \left\{\lambda_{A}(z \diamond x) \diamond(z \diamond \text { ? }), \lambda_{A}(\text { ? })\right\} \\
& \geq \min \left\{e \cdot \lambda_{A}(z \diamond x) \diamond(z \diamond \text { ? }), \text { e. } \lambda_{A}(\text { ? })\right\} \\
& \left(\lambda_{\mathrm{A}}\right)_{\mathrm{e}}^{\mathrm{M}}(\mathrm{x})=\min \left\{\left(\lambda_{\mathrm{A}}\right)_{\mathrm{e}}^{\mathrm{M}}(\mathrm{z} \diamond \mathrm{x}) \diamond(\mathrm{z} \diamond \text { ? }),\left(\lambda_{\mathrm{A}}\right)_{\mathrm{e}}^{\mathrm{M}}(\text { ? })\right\} \\
& \text { And, } \\
& \left(\xi_{\mathrm{A}}\right)_{\mathrm{e}}^{\mathrm{M}}(0)=\text { e. } \xi_{\mathrm{A}}(0) \leq \text { e. } \xi_{\mathrm{A}}(\mathrm{x}) \\
& \left(\xi_{\mathrm{A}}\right)_{\mathrm{e}}^{\mathrm{M}}=\left(\xi_{\mathrm{A}}\right)_{\mathrm{e}}^{\mathrm{M}}(\mathrm{x}) \\
& \left(\xi_{\mathrm{A}}\right)_{\mathrm{e}}^{\mathrm{M}}(\mathrm{x})=\mathrm{e} . \xi_{\mathrm{A}}(\mathrm{x}) \\
& \leq \text { e. } \max \left\{\xi_{\mathrm{A}}(\mathrm{z} \diamond \mathrm{x}) \diamond(\mathrm{z} \diamond \text { 目 }), \xi_{\mathrm{A}}(\text { 囵 })\right\} \\
& \leq \max \left\{e \cdot \xi_{A}(z \diamond x) \diamond(z \diamond \text { ? }), e . \xi_{A}(\text { 目 })\right\} \\
& \left(\xi_{\mathrm{A}}\right)_{\mathrm{e}}^{\mathrm{M}}(\mathrm{x})=\max \left\{\left(\xi_{\mathrm{A}}\right)_{\mathrm{e}}^{\mathrm{M}}(\mathrm{z} \diamond \mathrm{x}) \diamond\left(\mathrm{z} \diamond \text { 目), }\left(\xi_{\mathrm{A}}\right)_{\mathrm{e}}^{\mathrm{M}}(\text { ? })\right\}\right.
\end{aligned}
$$

Hence $A_{e}^{\mathrm{M}}=\left(\lambda_{\mathrm{A}}\right)_{\mathrm{e}}^{\mathrm{M}},\left(\xi_{\mathrm{A}}\right)_{\mathrm{e}}^{\mathrm{M}}$ is an IF_ INK-ideal of $K^{\prime}$.

\section{CONCLUSION}

In this paper, the conception of IFTR of IF_ INK_ I in INK-algebra are acquaint with and examined around of their valuable assets. We have given away that the IF a-translation of a intuitionistic $\mathrm{F}_{-} \mathrm{INK}_{-} \mathrm{I}$ is a intuitionistic $\mathrm{F}_{-}$ INK_I extension nevertheless the converse is not one true 


\section{REFERENCES}

1. K. Iseki and S. Tanaka, An introduction to the theory of BCK algebras, Math Japonica 1978;23:1- 20.

2. K. Iseki, On BCI-algebras, Math. Seminar Notes 1980;8: $125-130$.

3. K young Ja Lee, Young Bae Jun, and Myung Im Doh, Fuzzy Translations and Fuzzy Multiplications Of BCK/BCI- Algebras, Commun. Korean Math. Soc 2009; 24(3):353-360.

4. L.A. Zadeh, Fuzzy sets, Information and Control,1965; 338- 353

5. M. Kaviyarasu and K. Indhira, Fuzzy Subalgebras and Fuzzy K-ideals in INK-Algebras ,International Journal of Pure and Applied Mathematics2017; 113 (6): 47-55.

6. M. Kaviyarasu and K. Indhira, On intuitionistic fuzzy INK-ideals of INK-algebras, IOP Conference Series: Materials Science and Engineering 2017, 263(4).

7. M. Kaviyarasu and K. Indhira, Review on BCI/BCK-Algebras and Development, International Journal of Pure and Applied Mathematics 2017; 117(11): 377-383.

8. Ahmad, B, 'Fuzzy BCI-algebra', The Journal of Fuzzy Mathematics 1993; 1(2):445-452. 